Advancing Computing as a Science \& Profession

\title{
AISec'15
}

\section{Proceedings of the 8th ACM Workshop on Artificial Intelligence and Security}

Sponsored by:

\section{ACM SIGSAC}

Co-Located with:

CCS 2015 
Association for

Computing Machinery

Advancing Computing as a Science \& Profession

\section{The Association for Computing Machinery 2 Penn Plaza, Suite 701 New York, New York 10121-0701}

Copyright (C) 2015 by the Association for Computing Machinery, Inc. (ACM). Permission to make digital or hard copies of portions of this work for personal or classroom use is granted without fee provided that copies are not made or distributed for profit or commercial advantage and that copies bear this notice and the full citation on the first page. Copyright for components of this work owned by others than ACM must be honored. Abstracting with credit is permitted. To copy otherwise, to republish, to post on servers or to redistribute to lists, requires prior specific permission and/or a fee. Request permission to republish from: permissions@acm.org or Fax +1 (212) 869-0481.

For other copying of articles that carry a code at the bottom of the first or last page, copying is permitted provided that the per-copy fee indicated in the code is paid through www.copyright.com.

Notice to Past Authors of ACM-Published Articles

ACM intends to create a complete electronic archive of all articles and/or other material previously published by ACM. If you have written a work that has been previously published by ACM in any journal or conference proceedings prior to 1978, or any SIG Newsletter at any time, and you do NOT want this work to appear in the ACM Digital Library, please inform permissions@acm.org, stating the title of the work, the author(s), and where and when published.

ISBN: 978-1-4503-3826-4

Additional copies may be ordered prepaid from:

\section{ACM Order Department}

PO Box 30777

New York, NY 10087-0777, USA

Phone: 1-800-342-6626 (USA and Canada)

$+1-212-626-0500$ (Global)

Fax: +1-212-944-1318

E-mail: acmhelp@acm.org

Hours of Operation: 8:30 am - 4:30 pm ET

Printed in the USA 


\section{Foreword}

It is our great pleasure to welcome you to the 2015 ACM Workshop Artificial Intelligence and Security (AISec 2015) - the eighth annual workshop addressing technologies that fuse intelligent systems into computer security applications and the implications of these approaches. The papers to be presented in this year's program span topics ranging from adversarial learning, detecting fake OSN accounts, malware classification to privacy preserving data processing and game theoretic techniques in adversarial learning. The workshop's aim is to advance research at the intersection of artificial intelligence, machine learning, privacy and security. In particular, AISec gives researchers and practitioners working within one or more of those fields a platform for interdisciplinary discussion, which would otherwise be lacking. Hopefully, the workshop will lead to a high degree of cross-pollination between groups working across these areas. We are delighted to again be co-located with the premier ACM Computer and Communication Security (CCS 2015) conference.

This year we had 25 submissions from Asia, Europe and North America. After a rigorous reviewing process, involving at 2-3 referees per paper, 11 papers were accepted for presentation at the workshop, including presentation-only papers. Apart from the main program, there will also be a keynote speech by Pratyusa Manadhata titled "Machine Learning for Enterprise Security".

Organizing AISec 2015 was a team effort made possible by colleagues from institutions around the world. We sincerely thank the AISec program committee and external reviewers for their invaluable efforts towards guaranteeing a strong program, and the authors of papers who provided us with such excellent material. Their high caliber submissions and the thoughtful feedback from the reviewers continue to ensure the success of the ACM AISec workshop series. Finally, we wish to thank the ACM CCS organizers, particularly the workshop chairs Xiaofeng Wang and Kui Ren and CCS General Chair Indrajit Ray, for providing us with a suitable forum and for their continued support of this workshop.

We believe that the workshop program will be interesting not only for researchers working in the field, but also for practitioners and academics in other areas in security. We look forward to seeing you all in Denver this year.

$\begin{array}{lll}\text { Christos Dimitrakakis } & \text { Aikaterini Mitrokotsa } & \text { Arunesh Sinha } \\ \text { AISec'15 Program Chair } & \text { AISec'15 Program Chair } & \text { AISec'15 Program Chair } \\ \text { Chalmers University of } & \text { Chalmers University of } & \text { University of Southern } \\ \text { Technology, Sweden } & \text { Technology, Sweden } & \text { California, USA } \\ & & \\ & \text { Indrajit Ray } & \\ & \text { CCS'15 General Chair } \\ & \text { Colorado State University, USA }\end{array}$




\section{Table of Contents}

\section{Session: Keynote Address}

- Machine Learning for Enterprise Security 1 Pratyusa K. Manadhata (Hewlett-Packard Laboratories)

\section{Session: Privacy, Learning and Security}

- Fast, Privacy Preserving Linear Regression over Distributed Datasets based on Pre-Distributed Data

Martine De Cock (University of Washington), Rafael Dowsley (Karlsruhe Institute of Technology),

Anderson C. A. Nascimento, Stacey C. Newman (University of Washington)

- Differential Privacy for Classifier Evaluation ... 15

Kendrick Boyd, Eric Lantz, David Page (University of Wisconsin-Madison)

- Subsampled Exponential Mechanism: Differential Privacy in Large Output Spaces Eric Lantz, Kendrick Boyd, David Page (University of Wisconsin-Madison)

\section{Session: Malware and Malicious Activity}

- Malicious Behavior Detection using Windows Audit Logs

Konstantin Berlin, David Slater, Joshua Saxe (Invincea Labs, LLC)

- Better Malware Ground Truth: Techniques for Weighting Anti-Virus Vendor Labels 45

Alex Kantchelian (University of California, Berkeley),

Michael Carl Tschantz (International Computer Science Institute),

Sadia Afroz, Brad Miller, Vaishaal Shankar (University of California, Berkeley),

Rekha Bachwani (Netflix), Anthony D. Joseph (University of California, Berkeley),

J. D. Tygar (University of California, Berkeley)

- Remote Operating System Classification over IPv6

David Fifield (University of California, Berkeley),

Alexandru Geana (Technische Universiteit Eindhoven / Fox-IT, Delft),

Luis MartinGarcia (ETSIT, Polytechnic University of Madrid), Mathias Morbitzer (Fox-IT, Delft),

J. D. Tygar (University of California, Berkeley)

\section{Session: Adversarial Learning and Social Networks}

- Automated Attacks on Compression-Based Classifiers

Igor Burago (University of California, Irvine), Daniel Lowd (University of Oregon)

- Thwarting Fake OSN Accounts by Predicting their Victims

Yazan Boshmaf, Matei Ripeanu, Konstantin Beznosov (University of British Columbia),

Elizeu Santos-Neto (Google, Inc.)

- Detecting Clusters of Fake Accounts in Online Social Networks

Cao Xiao (University of Washington and LinkedIn Corporation),

David Mandell Freeman (LinkedIn Corporation), Theodore Hwa (LinkedIn Corporation) 


\section{AISec 2015 Workshop Organization}

General Chair: Indrajit Ray (Colorado State University, USA)

Workshop Chairs: Xiaofeng Wang (Indiana University, USA)

Kui Ren (SUNY Buffalo, USA)

Program Chairs: Christos Dimitrakakis (Chalmers University of Technology, Sweden) Aikaterini Mitrokotsa (Chalmers University of Technology, Sweden)

Arunesh Sinha (University of Southern California, USA)

Program Committee: Battista Biggio (University of Cagliari, Italy)

Michael Brückner (Amazon.com Inc, Germany)

Alvaro Cardenas (University of Texas at Dallas, TX, USA)

Kamalika Chaudhuri (University of California at San Diego, CA, USA)

Anupam Datta (Carnegie Mellon University, PA, USA)

David Freeman (LinkedIn Corporation, USA)

Joseph Halpern (Cornell University, NY, USA)

Alex Kantchelian (UC Berkeley, CA, USA)

Pavel Laskov (University of Tübingen, Germany)

Daniel Lowd (University of Oregon, OR, USA)

Pratyusa Manadhata (HP Labs, USA)

Blaine Nelson (University of Potsdam, Germany)

Sai Teja Peddinti (Google Inc., USA)

Roberto Perdisci (University of Georgia, GA, USA)

Konrad Rieck (University of Göttingen, Germany)

Fabio Roli (University of Cagliari, Italy)

Benjamin Rubinstein (University of Melbourne, Australia)

Robin Sommer (ICSI and LBNL, CA, USA)

Michael Tschantz (ICSI, Berkeley, CA, USA)

Doug Tygar (UC Berkeley, CA, USA)

Shobha Venkataraman (AT\&T Research, USA)

Additional Reviewers: Jeremiah Blocki ((Carnegie Mellon University, PA, USA)

Amit Datta (Carnegie Mellon University, PA, USA)

Samuel Jenkins (University of Melbourne, Australia)

Yuping Li (Kansas State University, USA)

Davide Maiorca ((University of Cagliari, Italy)

Miranda Mowbray (HP Labs, Bristol, UK)

Shayak Sen (Carnegie Mellon University, PA, USA)

Sponsor:

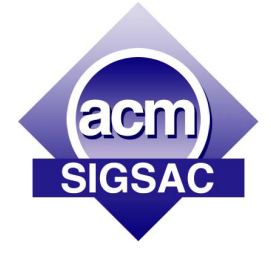

